\title{
Balanço energético na produção de feno de alfafa em sistema intensivo de produção de leite ${ }^{1}$
}

\author{
Energy balance in alfalfa hay production in a intensive system of milk production
}

\author{
Alessandro Torres Campos ${ }^{2}$ José Roberto Corrêa Saglietti ${ }^{3}$ Osmar de Carvalho Bueno ${ }^{4}$ \\ Aloísio Torres de Campos $^{5}$ Elcio Silvério Klosowski ${ }^{6}$ Eliane Gasparino $^{7}$
}

\section{RESUMO}

\begin{abstract}
O trabalho teve como objetivo estimar o balanço energético para a produção do feno de alfafa a partir de dados coletados durante sete anos em um Sistema Intensivo de Produção de Leite, localizado na região de Coronel Pacheco, MG. Para a confecção da matriz energética, foram consideradas as categorias de energia direta e indireta. As entradas de energia ocorridas na implantação $e$ estabelecimento da cultura foram diluídas nos anos considerados como vida útil. A energia direta, relativa aos insumos, teve maior participação que a indireta, sendo os percentuais encontrados de 93,05 e 6,95, respectivamente. $O$ principal consumidor de energia indireta foi o trator, que aciona todos os implementos, seguido pelo equipamento de irrigação. A eficiência energética estimada foi de 3,19. A maioria da energia direta utilizada foi aquela derivada de petróleo, na forma de combustível (óleo diesel). A utilização de adubo orgânico, de cama de frango, promoveu uma relativa redução no consumo de energia advinda de fertilizante derivado do petróleo. Com os resultados obtidos, pode-se concluir que, apesar das diversas fontes de consumo energético, a produção de feno de alfafa é sustentável e eficiente na conversão energética.
\end{abstract}

Palavras-chave: eficiência energética, sustentabilidade da agricultura, fenação.

\section{ABSTRACT}

The aim of this work was to evaluate the energy balance in the alfalfa hay production based on seven years data from an Intensive System of Milk Production, located in the region of Coronel Pacheco/MG Brazil. To create the energy matrix determination, the categories of direct and indirect energy were considered. The energy occurred in the implanting and establishing of the crop were distributed along the years considered as useful life. The direct energy, relative to the inputs, had larger participation than the indirect one, being the percentiles found of 93.05 and 6.95, respectively. The main consumer of indirect energy was the tractor, which turns on all the implements, followed by the irrigation system. The estimated energy efficiency by the process was of 3.19. Most of the direct energy used was derived from fossil resources, in the form of fuel (diesel). The use of organic fertilizer, poultry litter, promoted a relative reduction in the consumption of energy originated from fertilizer derived from fossil resources. With the obtained results, it was concluded that, despite the several sources of energy consumption, the production of alfalfa hay is sustainable and efficient in the energy conversion.

Key words: energy efficiency, hay production, sustainability of the agriculture.

\section{INTRODUÇÃO}

Sendo utilizada para animais de elevada exigência nutricional, a alfafa é mais difundida em países de clima temperado. Tanto para a venda como para consumo na fazenda, a fenação de alfafa é importante para conservar seu valor nutritivo, com máximo aproveitamento na época em que seu desenvolvimento não é tão vigoroso (TONUS, 1999). PAIM (1994) relata que exceção feita à Região Sul, as

${ }^{1}$ Parte da Tese de Doutorado apresentada pelo primeiro autor à Faculdade de Ciências Agronômicas, Universidade do Estado de São Paulo (UNESP).

${ }^{2}$ Engenheiro Agrícola, Doutor, Professor, Centro de Ciências Agrárias, UNIOESTE. Rua Pernambuco, 1777, 85960-000, Marechal Cândido Rondon, PR. E-mail: atcampos@unioeste.br (Coordenador do Grupo de Pesquisas em Ambiência do Oeste do Paraná - GPEA). ${ }^{3}$ Físico Doutor, Professor, Faculdade de Ciências Agronômicas, UNESP, Botucatu, SP.

${ }^{4}$ Engenheiro Agrônomo, Doutor, Professor, Faculdade de Ciências Agronômicas, UNESP, Botucatu, SP.

${ }^{5}$ Engenheiro Agrônomo, Doutor, Pesquisador, Embrapa Gado de Leite, Juiz de Fora, MG, (Membro do GPEA).

${ }^{6}$ Engenheiro Agrônomo, Doutor, Professor, Centro de Ciências Agrárias, UNIOESTE, (Membro do GPEA).

${ }^{7}$ Zootecnista, Doutora, Professora, Centro de Ciências Agrárias, UNIOESTE, (Membro do GPTEA). 
leguminosas forrageiras são pouco usadas em função da filosofia de buscar material para a condição ambiente. Neste âmbito, estudos de viabilidade técnica, econômica e ambiental, sob o ponto de vista de sustentabilidade, são interessantes como forma de fornecimento de subsídios para o incentivo ao aumento de produção desta forrageira no Brasil. Uma das metodologias mais empregadas para a avaliação da sustentabilidade de sistemas agrícolas tem sido o balanço energético. Como salienta ULBANERE (1988), com a crescente crise de petróleo, além do balanço contábil, passou a ter importância também o balanço energético, que é definido como um instrumento destinado a contabilizar as energias produzidas e as consumidas em um determinado sistema de produção. Seu objetivo principal é traduzir em unidades ou equivalentes energéticos os fatores de produção e os consumos intermediários, possibilitando a construção de indicadores comparáveis entre si, que permitam a intervenção no sistema produtivo visando melhorar sua eficiência (BUNEO et al., 2000).

Uma das maiores dificuldades na montagem das matrizes energéticas é a delimitação do sistema a ser estudado. Vários estudos têm apresentado dados baseados em estatísticas com limitações do espaço físico de grande amplitude, sendo alguns de nível estadual ou nacional. ZUCCHETTO \& JANSSON (1979) argumentam que estatísticas baseadas em médias de tais amplitudes dissimulam diferenças regionais, deixando de computar processos de agregação. Os estudos de casos com delimitação em sistemas fechados, com maior utilização de informações medidas in loco do que dependentes de estatísticas, médias amplas ou conjecturações, têm a importância de fornecer subsídios de maior exatidão para estudos de proporções mais amplas, em termos de delimitação física (CAMPOS, 2001).

O presente trabalho teve como objetivo estimar o balanço energético relativo à produção de feno de alfafa, em um Sistema Intensivo de Produção de Leite.

\section{MATERIAL E MÉTODOS}

No presente trabalho, foram utilizados dados da produção de feno de alfafa, do Sistema Intensivo de Produção de Leite (SIPL), da Embrapa Gado de Leite, em Coronel Pacheco-MG. A confecção da matriz energética foi iniciada pela definição do agrossistema e área, sendo caracterizado o período enfocado, detalhando o itinerário técnico. Foram elaboradas as rubricas operacionais, transformando os itens que compõem a matriz de exigência física em coeficientes energéticos (BUENO et al., 2000). O rendimento do sistema é dado pela relação (CARVALHO, 1980): Produção Utilizada pelo Homem/ "Inputs" Energéticos não Gratuitos. Neste trabalho, a "Produção Utilizada pelo Homem" é equivalente àquela energia disponível no feno, a ser fornecido aos animais. Como energia indireta, foi considerada aquela empregada na fabricação de maquinário, construções e instalações, sistemas de irrigação e outros "inputs" necessários à produção (CLEVELAND, 1995). Para o cálculo, foi utilizada metodologia desenvolvida por DOERING III et al. (1977), para quantificar os "inputs" de energia indireta utilizadas no sistema delimitado, através da depreciação energética, segundo os dias de utilização e em função da vida útil dos equipamentos. Como energia direta foram considerados: combustíveis fósseis, lubrificantes, fertilizantes e calcário, micronutrientes, herbicidas, diretamente consumidos no sistema agrícola (BONNY, 1993).

Para a condução da cultura da alfafa, a Embrapa Gado de Leite utilizou uma área de 3,3ha, no período de 1989 até 1995 . A correção do solo foi realizada segundo recomendações baseadas na análise química do solo, que apresentou os seguintes resultados: $\mathrm{pH}$ em água $(1: 2,5)=4,85, \mathrm{P}=16,75, \mathrm{~K}=$ 47,25, expressos em ppm; $\mathrm{Ca}=2,95, \mathrm{Mg}=1,3$ e $\mathrm{Al}=$ 0,675 , expressos em meq. $100 \mathrm{~cm}^{-3}$. Aplicou-se por ocasião do plantio, $600 \mathrm{~kg}$ ha $^{-1}$ de superfosfato simples, equivalente a $120 \mathrm{~kg}$ de $\mathrm{P}_{2} \mathrm{O}_{5}$, mais $10 \mathrm{t} \cdot \mathrm{ha}^{-1}$ de cama de aviário. As percentagens disponíveis de $\mathrm{N}, \mathrm{P}_{2} \mathrm{O}_{5}$ e $\mathrm{K}_{2} \mathrm{O}$ na cama de aviário consideradas foram de 3,5; 4,0 e 3,0; respectivamente (AVILA et al., 1992). O processo de preparo do solo e semeadura ocorreu na seguinte sequiência: distribuição da metade do calcário, com equipamento próprio, aração (com arado de aiveca), segunda distribuição de calcário, distribuição de esterco com carreta, distribuição do superfosfato simples, gradagem (aradora), aplicação do herbicida com pulverizador de barra, incorporação do herbicida por meio de gradagem leve. As sementes utilizadas foram inoculadas com Rhizobium melioti, semeadas em operação mecanizada, a uma taxa de 15 a $17 \mathrm{~kg}$ sementes viáveis por hectare. Na adubação de plantio foi utilizado: superfosfato simples $\left(500 \mathrm{~kg} \cdot \mathrm{ha}^{-1}\right)$; cloreto de potássio $\left(120 \mathrm{~kg} \cdot \mathrm{ha}^{-1}\right)$; nitrocálcio $\left(300 \mathrm{~kg} \cdot \mathrm{ha}^{-1}\right)$; fertilizante FTE BR 16 ("Fritted Trace Elements" composto por $1,5 \%$ de $\mathrm{B}, 3,5 \%$ de $\mathrm{Cu}, 0,4 \%$ de $\mathrm{Mo} \mathrm{e}$ $3,5 \%$ de $\mathrm{Zn})\left(50 \mathrm{~kg} \cdot \mathrm{ha}^{-1}\right)$. No estabelecimento da cultura, foi aplicado herbicida Eradicane (EPTC) PPI, à taxa de 7,0 1/ha. No pós-plantio foi aplicado Poast + Assist à taxa de 1,5L.ha-1 ou os seguintes: Bentazon, Fluazifopbutil, Hloxyfop-metil e Sethoxydin. Na adubação de 
manutenção foi utilizado $60 \mathrm{~kg} \cdot \mathrm{ha}^{-1}$ de Superfosfato simples, e 120kg.ha- ${ }^{-1}$ de Cloreto de Potássio, aplicados a cada dois cortes. Duas vezes por ano foram aplicados os micronutrientes. O sistema de irrigação por aspersão teve um turno de rega semanal, do plantio aos dois primeiros meses após a germinação, e, em média, um turno a cada 15 dias, no período de manutenção da cultura. Os cortes destinados à produção de feno (média de 10 por ano), foram realizados por segadora de dois discos, tracionada por trator Massey Fergunson 275 Simples de 75CV. Após cada corte, a forragem foi mantida no próprio local, sendo espalhada, enleirada e revolvida, sendo utilizado ancinho.

\section{Coeficientes Energéticos/Conversão Energética}

Trabalho Humano: Foi considerada a energia advinda dos trabalhadores efetivamente utilizada na produção de feno, ponderada pela carga horária dedicada a esta atividade, através do coeficiente energético de $386,40 \mathrm{~kJ} . \mathrm{h}^{-1}$ de trabalho (CARVALHO et al., 1974). Instalações: A composição energética do Galpão de Armazenamento de Feno foi estimada através dos coeficientes energéticos apresentados por FERNANDES \& SOUZA, (1982). Insumos: Os valores energéticos dos elementos, foram obtidos, via de regra, através da multiplicação de sua massa (kg) ou volume (litros) pelo conteúdo energético específico correspondente, apresentado pela literatura, ponderando através de seu nível de utilização por hectare, na cultura. Material de Propagação: A energia para a produção das sementes de alfafa, segundo HEICHEL (1980), é de 182,51kJ.kg-1 . Através de procedimento de estimativa de custo de energia fóssil para produção de material de propagação, baseado nos custos econômicos, proposto por HEICHEL (1980) foi determinado o custo energético por $\mathrm{kg}$ do produto (inoculante) de $691.481,31 \mathrm{~kJ}$. Fertilizantes e Corretivos: Os valores adotados para os elementos são aqueles informados por PELLIZZI (1992): $\mathrm{N}=73 \mathrm{MJ} \cdot \mathrm{kg}^{-1} ; \mathrm{P}_{2} \mathrm{O}_{5}=13 \mathrm{MJ} \cdot \mathrm{kg}^{-1} ; \mathrm{K}_{2} \mathrm{O}=9 \mathrm{MJ} \cdot \mathrm{kg}^{-1}$. Multiplicaram-se as quantidades efetivas dos elementos ativos $\left(\mathrm{N}, \mathrm{P}_{2} \mathrm{O}_{5}\right.$ e $\mathrm{K}_{2} \mathrm{O}$, em $\mathrm{kg}$ ) pelo valor energético correspondente. Para os micronutrientes, foi adotada a metodologia de determinação do coeficiente energético baseada na intensidade energética do produto nacional bruto, em $\mathrm{kJ}$ por valor monetário, preconizada por HEICHEL (1980). A energia contida no calcário foi obtida a partir da equação proposta por MACEDÔNIO \& PICCHIONI (1985), que relaciona o valor energético embutido no produto e o consumo de energia no transporte: $(40.938+228 \times \mathrm{d}) \times$ $4,184 \times 10^{-3}\left[\mathrm{~kJ} \cdot \mathrm{kg}^{-1}\right]$; sendo d a distância percorrida, em km. O coeficiente energético calculado para o calcário foi de $229 \mathrm{~kJ} \mathrm{~kg}^{-1}$. Herbicidas: os coeficientes energéticos são informados por CLEMENTS et al. (1995), considerando, ainda, uma energia adicional, de $20 \mathrm{MJ} . \mathrm{kg}^{-1}$ de ingrediente ativo, necessária para a formulação do herbicida (Green, apud CLEMENTS et al., 1995). O teor de ingrediente ativo é informado por LACA-BUENDIA et al. (1985). Máquinas e Equipamentos: Utilizou-se a metodologia desenvolvida por DOERING III et al. (1977), empregada por diversos autores (BEBER, 1989; SERRA et al., 1979; ULBANERE, 1988; entre outros), baseada na depreciação energética, que a partir da massa das máquinas, consiste em depreciá-las durante sua vida útil. Os coeficientes adotados foram (MACEDÔNIO \& PICCHIONI, 1985): trator $=69,83 \mathrm{MJ} \cdot \mathrm{kg}^{-1}$; outros equipamentos $=57,20 \mathrm{MJ} \cdot \mathrm{kg}^{-1}$; pulverizador costal $=$ $72 \mathrm{MJ} . \mathrm{kg}^{-1}$ (BANSAL et al., 1988). Irrigação: O consumo energético para o funcionamento dos equipamentos é de $92 \mathrm{~kJ} . \mathrm{h}^{-1} \cdot \mathrm{m}^{-1}$, e $2,40 \mathrm{MJ} \cdot \mathrm{h}^{-1}$ para o bombeamento (TSATSARELIS, 1993). Combustiveis e Lubrificantes: Constituem, basicamente, óleo diesel, óleo lubrificante e graxa, com coeficientes energéticos de: $43.930 \mathrm{~kJ}^{-1} \mathrm{1}^{-1}, 43.908 \mathrm{~kJ} .1^{-1}$ e $49.224 \mathrm{~kJ} . \mathrm{kg}^{-1}$, respectivamente (SERRA et al., 1979).

\section{RESULTADOS E DISCUSSÃO}

A produtividade média de alfafa foi de 17,5t MS.ha ${ }^{-1}$.ano ${ }^{-1}$. A etapa de implantação requereu vastas operações, porém, o consumo energético despendido nesta fase teve seu valor distribuído entre os sete anos de utilização da cultura. A tabela 1 mostra a participação das diversas máquinas, equipamentos e estruturas envolvidas na composição da energia indireta. Entre os implementos, somente a carreta teve emprego nas três fases, com maior participação na etapa de fenação. Sendo o trator utilizado como acionador da maioria dos equipamentos, sua participação na composição dos consumos energéticos indiretos foi muito maior que os demais itens. Os implementos de revolvimento do solo, apesar de demandarem apreciáveis quantidades de energia, tiveram menor participação relativa, devido ao fato de seus valores estarem diluídos nos sete anos de vida da cultura. O cômputo geral da energia empregada na produção de feno, nas formas direta e indireta, é sintetizado na tabela 2. O maior consumo de energia observado foi da categoria direta com 93,05\% do total, sendo $6,95 \%$ para a indireta. Houve uma preponderância no uso de energia fóssil, através do combustível utilizado nas operações mecanizadas, desenvolvidas por meio de trator. Itens como sementes 
Tabela 1 - Composição da energia indireta aplicada em equipamentos e estruturas para alfafa

\begin{tabular}{|c|c|c|c|c|}
\hline \multirow{2}{*}{ Equipamento } & \multicolumn{2}{|c|}{$\begin{array}{l}\text { Participação como energia indireta nas fases } \\
\qquad(\mathrm{MJ} / \mathrm{ha} / \mathrm{ano})\end{array}$} & \multicolumn{2}{|c|}{ Total } \\
\hline & $\begin{array}{l}\text { Implantação da } \\
\text { Cultura }\end{array}$ & Manutenção Anual & Fenação & (MJ/ha/ano) \\
\hline Trator modelo MF 275 simples & 89,28 & 191,63 & 2629,10 & $2.910,00$ \\
\hline Subsolador & 0,58 & - & - & 0,58 \\
\hline Arado de 3 aivecas reversíveis & 5,89 & - & - & 5,89 \\
\hline Pulverizador de barra & 2,68 & 46,50 & - & 49,18 \\
\hline Grade aradora & 4,54 & - & - & 4,54 \\
\hline Grade niveladora & 2,81 & - & - & 2,81 \\
\hline Enxada rotativa & 21,11 & - & - & 21,11 \\
\hline Semeadora adubadora & 3,88 & - & - & 3,88 \\
\hline Carreta agrícola de madeira & 2,39 & 14,93 & 137,31 & 154,62 \\
\hline Distribuidor de calcário & 3,05 & - & - & 3,05 \\
\hline Equipamento de irrigação & 15,25 & 534,27 & - & 549,52 \\
\hline Segadora & - & - & 121,80 & 121,80 \\
\hline Ancinho enleirador & - & - & 103,31 & 103,31 \\
\hline Enfardadora & - & - & 215,80 & 215,80 \\
\hline Galpão de armazenamento & - & - & 86,11 & 86,11 \\
\hline TOTAL & 151,47 & 787,32 & $3.293,42$ & $4.232,20$ \\
\hline
\end{tabular}

Tabela 2 - Balanço energético consolidado para a produção de feno a partir da cultura da alfafa no SIPL/EMBRAPA - Gado de Leite

\begin{tabular}{|c|c|c|}
\hline Fonte de Consumo (“inputs") & Consumo de Energia MJ/ha/ano & Porcentagem do Total \\
\hline \multicolumn{3}{|l|}{ ENERGIA INDIRETA } \\
\hline \multicolumn{3}{|l|}{ Máquinas e Equipamentos } \\
\hline Trator & $2.910,00$ & 4,78 \\
\hline Implementos & 686,58 & 1,13 \\
\hline Construções e Instalações & 86,11 & 0,14 \\
\hline Equipamento de Iirrigação & 549,52 & 0,90 \\
\hline SUB-TOTAL - Energia Indireta & $4.232,20$ & 6,95 \\
\hline \multicolumn{3}{|l|}{ ENERGIA DIRETA } \\
\hline \multicolumn{3}{|l|}{ Fertilizantes } \\
\hline $\mathrm{N}$ & $4.275,71$ & 7,02 \\
\hline $\mathrm{P}_{2} \mathrm{O}_{5}$ & $1.931,43$ & 3,17 \\
\hline $\mathrm{K}_{2} \mathrm{O}$ & $3.718,29$ & 6,11 \\
\hline Micronutrientes & 446,86 & 0,73 \\
\hline Calcário & 245,68 & 0,40 \\
\hline \multicolumn{3}{|l|}{ Combustíveis e Lubrificantes } \\
\hline Óleo Diesel & $45.030,38$ & 73,94 \\
\hline Óleo Lubrificante & 569,45 & 0,94 \\
\hline Graxa & 103,30 & 0,17 \\
\hline Irrigação & 172,22 & 0,28 \\
\hline Herbicidas & 5,66 & 0,01 \\
\hline Sementes e Material de Propagação (Inoculante) & 17,24 & 0,03 \\
\hline Mão-de-obra & 150,88 & 0,25 \\
\hline SUB-TOTAL - Energia Direta & $56.667,11$ & 93,05 \\
\hline TOTAL & $60.899,31$ & 100,00 \\
\hline Energia Efetiva contida no Feno de Alfafa & $193.696,23$ & \\
\hline Eficiência Energética & 3,19 & \\
\hline
\end{tabular}

Ciência Rural, v. 34, n. 1, jan-fev, 2004. 
(incluindo o inoculante), mão-de-obra, irrigação, micronutrientes e os herbicidas, que são essenciais à cultura, apresentaram valores de consumo energético baixíssimos na composição da matriz (Tabela 2). Em segundo lugar no consumo energético, se apresentam os fertilizantes, que, de um gasto total de 9.925,43MJ.ha- ${ }^{-1}$. ano ${ }^{-1}$, dos quais $48,14 \%$ tiveram origem em material orgânico e $51,86 \%$ em derivados de petróleo, têm papel primordial na viabilidade da condução desta cultura. A figura 1 demonstra de forma mais clara as participações dos elementos, de origem fóssil ou orgânica, em que se percebe que a maior parte do $\mathrm{N}$ utilizado é advindo do esterco. Fontes de $\mathrm{N}$ somente foram utilizadas por ocasião da implantação da cultura. A contribuição energética mais intensa de elemento de origem fóssil foi para o $\mathrm{K}_{2} \mathrm{O}$, aplicado de forma separada, ou seja, sem uso de formulação composta com N. Desde os trabalhos pioneiros encontrados na literatura (PIMENTEL et al., 1973) remonta-se uma preocupação acerca da elevada carga energética encontrada no uso de $\mathrm{N}$ advindo de adubos minerais. Torna-se interessante a análise do comportamento de consumo de energia embutida em fertilizantes nitrogenados na cultura da alfafa sob dois aspectos: a) A alfafa tem a capacidade de fixar $\mathrm{N}$ do ar atmosférico, desta forma, o uso de fertilizantes nitrogenados só ocorre na implantação da cultura; b) O único fornecimento ocorrido, deste elemento, se caracterizou por uma preponderância de utilização de fonte orgânica, em detrimento do uso de derivados de petróleo. Estes fatos caracterizam um comportamento diferenciado da alfafa no uso de insumos energéticos na forma de adubo em relação às culturas amplamente estudadas no âmbito dos balanços de energia, e com maior divulgação, de uma forma geral, no meio científico.

Sendo o NDT médio, para a alfafa, de $60 \%$, e, tomando como base a produção média anual de matéria seca da cultura, tem-se que a produtividade média de nutrientes digestíveis totais foi de $10.500 \mathrm{~kg} \cdot \mathrm{ha}^{-1} \cdot \mathrm{ano}^{-1}$. Considerando-se o valor energético de $18.447,26 \mathrm{~kJ} \mathrm{~kg}^{-1}$ de NDT, depreende-se que a energia total embutida na alfafa produzida é de 193.696,23 MJ/ha/ano. A partir do valor total de energia gasto (tabela 2), conclui-se que a eficiência energética para a produção do feno foi de 3,19 . HEICHEL \& MARTIN (1980) obtiveram eficiência energética de 1,85 ("output" de energia digestível/ "input" de energia fóssil), na produção de feno de alfafa em uma área semiárida na região oriental do Colorado, com clima temperado; sendo que o maior consumidor de energia foi o combustível, e, em segundo, $\mathrm{o}_{2} \mathrm{O}_{5}$, não havendo utilização de N. Os autores não detalharam os métodos culturais, mas, segundo afirmam os mesmos, a eficiência energética desta cultura é extremamente dependente do ambiente de crescimento, das técnicas culturais, de manejo e colheita, que podem variar muito. O número possível de cortes anuais, de acordo com as características climáticas da região, assim como a quantidade e forma de operações mecanizadas na condução, colheita e fenação da alfafa são fatores que fazem com que haja uma grande variedade de resultados de eficiência energética, mesmo dentro de um mesmo país, para uma mesma época. Esta disparidade pode se acentuar para regiões distantes (como o Brasil e os Estados Unidos), principalmente para épocas diferentes. A participação energética do combustível, na formação do balanço, superou os dados constantes na literatura para outras culturas. QUESADA et al. (1987), por exemplo, informam participações de $47,6 \% ; 46,5 \%$ e $40,7 \%$, na energia advinda do óleo diesel, para as culturas de milho, soja e trigo, respectivamente, em um estudo no Rio Grande do Sul. Em todas as etapas, percebeu-se baixo consumo de energia na forma de trabalho humano, o que motiva autores, como ULBANERE (1988), a desconsiderar a utilização desta rubrica na contabilização do balanço energético. Para o presente trabalho, os valores baixos ocorreram devido às características da delimitação adotada para o sistema em estudo. Para sistemas mais amplos, pesquisas levando em consideração as apresentações de FLUCK (1981) poderiam propiciar consumos bem mais elevados para a mão-de-obra, devido àquela energia indireta implícita necessária para a manutenção do padrão de vida do trabalhador e sua família. No SIPL, foi utilizado adubo orgânico para a implantação da cultura da alfafa, o que promoveu

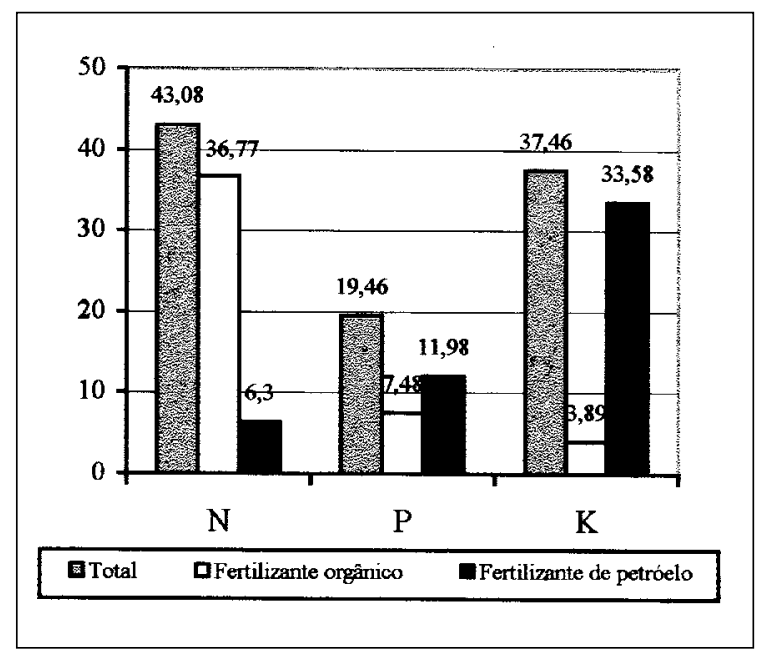

Figura 1 - Distribuição percentual da energia empregada em N ("N"), $\mathrm{P}_{2} \mathrm{O}_{5}$ ("P") e $\mathrm{K}_{2} \mathrm{O}$ (" $\mathrm{K}$ ") nos fertilizantes utilizados, de origem orgânica ou derivada de petróleo.

Ciência Rural, v. 34, n. 1, jan-fev, 2004. 
considerável aumento de utilização de energia não derivada do petróleo. Complementando, MELLO (1986) aponta que a incorporação de restos de culturas, resíduos vegetais e animais, tem custo energético relativamente reduzido, gerando resultados satisfatórios em fertilização, além de serem integrantes naturais de cadeias de ecossistemas.

\section{CONCLUSÕES}

Os itens irrigação, mudas, mão-de-obra e herbicidas apresentaram reduzido consumo de energia e o maior consumidor de energia indireta foi o trator, seguido do equipamento de irrigação. Os implementos que demandam mais energia indireta foram aqueles usados na fenação. $\mathrm{O}$ consumo total de energia para a produção do feno é de $60.899,31 \mathrm{MJ} \cdot \mathrm{ha}^{-1} \cdot \mathrm{ano}^{-1}$, sendo $6,95 \%$ na forma indireta e $93,05 \%$ na forma direta, e a eficiência energética para a produção do feno é de 3,19 . Os derivados de petróleo são os maiores consumidores de energia, sendo o óleo diesel responsável por 73,94\% do consumo total. Apesar dos elevados valores absolutos de consumo energético, a produção se mostrou eficiente na utilização da energia empregada, evidenciando a sustentabilidade da produção.

\section{AGRADECIMENTO}

O primeiro autor agradece à CAPES pela concessão de bolsa de Doutorado.

\section{REFERÊNCIAS BIBLIOGRÁFICAS}

AVILA, V.S.; MAZZUCO, H.; FIGUEIREDO, E.A.P. Cama de aviário: materiais, reutilização, uso como alimento e fertilizante. Concórdia : EMBRAPACNPSA, 1992. 38p. (EMBRAPA-CNPSA. Circular Técnica, 16).

BANSAL, R.K.; KSHIRSAGAR, K.G.; SANGLE, R.D. Efficient utilization of energy with an improved farming system for selected semi-arid tropics. Agriculture, Ecosystems and Environment, Amsterdam, v.24, n.4, p.381-394, 1988 .

BEBER, J.A.C. Eficiência energética e processos de produção em pequenas propriedades rurais, Agudo, RS. 1989. 295f. Dissertação (Mestrado em Extensão Rural) Curso de Pós-graduação em Extensão Rural, Universidade Federal de Santa Maria.

BONNY, S. Is agriculture using more and more energy? A French case study. Agricultural Systems, Essex, UK, v.43, n.1, p.51-66, 1993.

BUENO, O.C.; CAMPOS, A.T.; CAMPOS, A.T. Balanço de energia e contabilização da radiação global: simulação e comparativo. In: Avances en ingenería agrícola. Buenos Aires : Facultad de Agronomia, 2000. p.477-482.

CAMPOS, A.T. Balanço energético relativo à produção de feno de "coast-cross" e alfafa em sistema intensivo de produção de leite. 2001. 236f. Tese (Doutorado em Energia na Agricultura) - Curso de Pós-graduação em Agronomia, Universidade Estadual Paulista.

CARVAlho, A. Análise eco-energética dos sistemas de produção agrícola da zona vitícola de Dois Portos (Torres Vedras). Lisboa : Fundação Calouste Gulbenkian, 1980. 44p.

CARVAlHO, A.; GONÇALVES, G.G.; RIBEIRO, J.J.C. Necessidades energéticas de trabalhadores rurais e agricultores na sub-região vitícola de Torres. Lisboa : Fundação Calouste Gulbenkian, 1974. 79p.

CLEMENTS, D.R. et al. Energy analysis of tillage and herbicide inputs in alternative weed management systems. Agriculture, Ecosystems and Environment, Amsterdam, v.52, n.2-3, p.119-128, 1995.

CLEVELAND, C.J. The direct and indirect use of fossil fuels and electricity in USA agriculture, 1910-1990. Agriculture, Ecosystems and Environment, Amsterdam, v.55, n.2, p.111-121, 1995.

DOERING III, O.C.; CONSIDINE, T.J.; HARLING, C.E. Accouting for tillage equipment and other machinery in agricultural energy analysis. Indiana, West Lafayette: Purdue University, 1977. (Agr. Exp. Sta. NSF/RA - 770128). $4 \mathrm{p}$.

FERNANDES, M.P.; SOUZA, A.M.T. Balanço energético o consumo de energia na construção civil. Revista Brasileira de Tecnologia, Brasília, v.13, n.3, p.22-36, jun./jul, 1982.

FLUCK, R.C. Net energy sequestered in agricultural labor. Transactions of the ASAE, St. Joseph, Michigan, v.24, n.6, p.1449-1455, 1981 .

HEICHEL, G.H. Assessing the fossil energy costs of propagating agricultural crops. In: PIMENTEL, D. Handbook of energy utilization in agriculture. Boca Raton : CRC, 1980. p.27-33.

HEICHEL, G.H.; MARTIN, N.P. Energy inputs and outputs for crop systems - alfalfa. In: PIMENTEL, D. Handbook of energy utilization in agriculture. Boca Raton : CRC, 1980. p.155-161.

LACA-BUENDIA, J.P. et al. Herbicidas recomendados para as principiais culturas do Estado de Minas Gerais. Informe Agropecuário, Belo Horizonte, v.11, n.127, p.32-65, jul, 1985 .

MACEDÔNIO, A.C.; PICCHIONI, S.A. Metodologia para o cálculo do consumo de energia fóssil no processo de produção agropecuária. v.1. Curitiba : Secretaria de Estado da Agricultura, 1985. V.1, 95p.

MELLO, R. de. Análise energética de agroecossistemas: o caso de Santa Catarina. 1986. 139f. Tese (Mestrado em Engenharia de Produção) - Curso de Pós-graduação em Engenharia, Universidade Federal de Santa Catarina. 
PAIM, N.R. Utilização e melhoramento da alfafa. In: WORKSHOP SOBRE O POTENCIAL FORRAGEIRO DA ALFAFA (Medicago sativa L.) NOS TRÓPICOS, 1., 1994, Juiz de Fora. Anais... Juiz de Fora : EMBRAPA-CNPGL. 1994. p.141-147.

PELLIZZI, G. Use of energy and labour in Italian agriculture. Journal of Agricultural Engineering Research, Silsoe Research Institute, v.52, n.2, p.111-119, 1992.

PIMENTEL, D. et al. Food production and the energy crises Science, N.Y. (USA), v.182, p.443-449, 1973.

QUESADA, G.M.; BEBER, J.A.C.; SOUZA, S.P. Balanços energéticos: uma proposta metodológica para o Rio Grande do Sul. Ciência e Cultura, São Paulo, v.39, n.1, p.20-28, jan, 1987 .

SERRA, G. et al. Avaliação da energia investida na fase agrícola de algumas culturas. Brasília : Secretaria de Tecnologia Industrial, 1979. 86p.
TONUS, M. Alfafa - o bom cultivo viabiliza seu uso em qualquer região. Balde branco, São Paulo, v.35 n.416, p.2833, jun. 1999

TSATSARELIS, C.A. Energy inputs and outputs for soft winter wheat production in Greece. Agriculture, Ecosystems and Environment, Amesterdam, v.43, n.2, p.109-118, 1993.

ULBANERE, R.C. Análise dos balanços energético e econômico relativa à produção e perda de grãos de milho no Estado de São Paulo. 1988. 127f. Tese (Doutorado em Energia na Agricultura) - Curso de Pósgraduação em Agronomia, Universidade Estadual Paulista.

ZUCCHETTO, J.; JANSSON, A-M. Total energy analysis of Gotland's agriculture: a northern temperature zone case study. Agro-ecosystems, Amsterdam, v.5, p.329-344, 1979. 